\title{
O papel do capital humano no crescimento - uma análise espacial para o Brasil ${ }^{\dagger}$
}

\author{
Ariene da Silva Salgueiro* \\ Luciano Nakabashi* \\ Diogo de Prince ${ }^{* * *}$
}

\begin{abstract}
RESUMO - O presente trabalho investiga o papel do espaço e dos fatores de produção no crescimento dos municípios brasileiros entre 1991 e 2000. O estudo abarca os modelos de Solow-Swan (1956) e Mankiw, Romer e Weil (MRW) (1992). Os resultados obtidos foram de que a presença de correlação espacial é confirmada pelo teste do I de Moran, e que os capitais físico e humano são relevantes para explicar o crescimento econômico.
\end{abstract}

Palavras-chave: Crescimento. Econometria espacial. Fatores de produção.

\section{INTRODUÇÃO}

A teoria sobre crescimento econômico apresenta uma extensa literatura, contemplada por diversas linhas de estudo. Um grande número de trabalhos trata dos determinantes do crescimento econômico e do diferencial de renda entre países e regiões. A inserção das teorias do capital humano, difusão de tecnologia e crescimento endógeno trouxeram contribuições relevantes no entendimento desses processos. Gradualmente, essas teorias têm sido testadas empiricamente, assim como métodos de estimação mais adequados têm sido empregados para a comparação de diferentes países ou regiões de um mesmo país, como a introdução do efeito espacial.

Em relação aos estudos empíricos que contemplam o efeito espacial no Brasil, muitos deles buscam tratar a questão da convergência de renda. Apesar desses estudos, há uma escassez de trabalhos para o caso brasileiro considerando o efeito espacial e o referencial teórico com base nas teorias de crescimento econômico citadas anteriormente.

\footnotetext{
† Os autores agradecem ao auxílio no desenvolvimento da base de dados prestado por Amauri de Souza Porto Junior, pelo auxílio na rotina por Roger Bivand, e também ao apoio financeiro concedido pela CAPES/REUNI. Cabe, entretanto eximi-los de qualquer responsabilidade por eventuais erros ou omissões.

* Economista pela Universidade Estadual Paulista Júlio de Mesquita Filho/Araraquara. Mestranda no Programa de Pós-Graduação em Desenvolvimento Econômico pela Universidade Federal do Paraná. Endereço eletrônico: arienesalgueiro@yahoo.com.br.

** Doutor em economia pela Universidade Federal de Minas Gerais. É professor do Departamento de Economia da Universidade Federal do Paraná e pesquisador do CNPq. Endereço eletrônico: luciano.nakabashi@gmail.com. *** Mestre pela Faculdade de Economia, Administração e Contabilidade de Ribeirão Preto/Universidade de São Paulo. Doutorando pela Escola de Economia de São Paulo/Fundação Getúlio Vargas. Endereço eletrônico: dioted@gmail.com.
} 
Sob o ponto de vista econométrico, é importante considerar o problema da correlação espacial, pois a sua omissão conduz à tendenciosidade dos estimadores das variâncias, o que invalida os testes de hipóteses. O uso de um modelo de econometria espacial apropriado possibilita levar em conta as externalidades espaciais não observáveis existentes no processo de crescimento.

Assim, a contribuição do presente estudo é a estimação de dois modelos de crescimento para os municípios brasileiros, levando em consideração a existência de dependência espacial entre eles. O modelo teórico tem como base a importância dos fatores de produção sobre o crescimento (SOLOW-SWAN, 1956; MANKIW; ROMER; WEIL, 1992). A análise baseia-se nos municípios brasileiros no período entre 1991 e 2000 . Adicionalmente, testa-se a hipótese da convergência considerando a questão espacial.

Além dessa introdução, este artigo está dividido em mais três seções. Na Seção 2, apresenta-se o modelo que serve de base para a análise empírica. Na terceira, as fontes de dados e a metodologia utilizada. E por fim na quarta, os resultados das estimações econométricas são apresentados e interpretados.

\section{MODELOS TEÓRICOS}

No presente estudo, pretendemos examinar os efeitos dos fatores de produção na determinação da renda. A base teórica encontra-se nos modelos de crescimento de Solow e MRW. A especificação da função renda $(Y)$ é a seguinte:

$$
Y(t)=K(t)^{\alpha} H(t)^{\beta}[A(t) L(t)]^{1-\alpha-\beta}
$$

onde $\alpha$ e $\beta$ são as elasticidades do produto em relação aos insumos. A inclusão da variável capital humano $(H)$ reduz o efeito da poupança e do crescimento populacional sobre a renda. Os demais fatores de produção são capital físico $(K)$, trabalho $(L)$ e tecnologia $(A)$. As participações do capital físico, humano e trabalho na renda são $\alpha, \beta$ e $(1-\alpha-\beta)$, respectivamente.

A partir da Equação (1), das suposições realizadas por Solow e MRW para as funções de acumulação do capital físico e humano, e considerando o estado estacionário, chegamos à seguinte equação:

$$
\ln y_{t}=\ln \left[\frac{Y_{t}}{L_{t}}\right]=\ln A_{0}+g t-\frac{\alpha+\beta}{1-\alpha-\beta} \ln (n+g+\delta)+\frac{\alpha}{1-\alpha-\beta} \ln \left(s_{k}\right)+\frac{\beta}{1-\alpha-\beta} \ln \left(s_{b}\right)
$$

onde $s_{b}$ representa a fração de renda investida em capital humano e $s_{k}$ a fração da renda investi- 
da em capital físico. Primeiramente, pode-se observar que uma maior taxa de investimento em capital físico e humano ou menor taxa de crescimento populacional levam a um nível de renda maior, no estado estacionário, constatando-se que há uma relação positiva entre renda per capita e capital humano. Suposições adicionais são de que o capital se deprecia a uma taxa constante $\delta$, a população cresce à taxa $n$ e a tecnologia a uma taxa constante e exógena $g$.

No tocante à convergência, os autores relaxam a hipótese de os países estarem no estado estacionário. Considerando o período de convergência, tem-se:

$$
\ln y_{t}=\left(1-e^{-\lambda t}\right) \ln \left(y^{*}\right)+e^{-\lambda t} \ln y_{0}
$$

onde: $\mathrm{y}^{*}$ é o nível de renda do estado estacionário em unidades efetivas de trabalho; $\lambda$ denota a taxa de convergência, entendida por $\lambda=(n+g+\delta)(1-\alpha-\beta)$. Substituindo $y^{*}$ e subtraindo $y_{0}$ de ambos os lados:

$$
\ln \left(\frac{y_{t}}{y_{0}}\right)=\left(1-e^{-\lambda t}\right)\left[-\ln \left(y_{0}\right)+\frac{\alpha}{1-\alpha-\beta} \ln \left(s_{k}\right)-\frac{\alpha+\beta}{1-\alpha-\beta} \ln (n+g+\delta)+\frac{\beta}{1-\alpha-\beta} \ln \left(s_{b}\right)+\ln A_{0}+g t\right]
$$

A variável y é o nível de renda por trabalho; $\lambda$ denota a taxa de convergência, entendida por: $\lambda=(n+g+\delta)(1-\alpha-\beta)$. Com a Equação (4), MRW concluem que ocorre convergência condicional a uma taxa próxima ao previsto pelo modelo de Solow, quando se considera o fator capital humano. Está é a equação que serve de base para a estimação econométrica no caso dos municípios brasileiros.

\section{FONTE DOS DADOS E METODOLOGIA}

\subsection{DADOS}

Os dados basicamente referem-se aos anos de 1991 e de 2000 para os municípios brasileiros $^{1}$. Os softwares utilizados na estimação dos modelos foram o Geoda, Ipeageo e R. A modificação geográfica do Brasil, ou seja, a criação de municípios, dificulta análises realizadas ao longo do tempo. Para resolver esse problema, utilizou-se o índice de organização da divisão territorial disponível pelo IBGE para agrupar os novos munícipios e obter dados comparáveis ao longo do tempo.

Após este reagrupamento, a construção das variáveis passa a ser mais simples. A força

1 Com exceção da Despesa de Capital - Investimento - Municipal - R\$, em que o período inicial utilizado corresponde ao ano de 1993. 
de trabalho do período inicial $\left(L_{0}\right)$ é medida pela população residente-total-habitante em 1991, já para o período $\left(L_{t}\right)$ final utiliza-se a média dos períodos inicial e final. Para a construção da força de trabalho basta tirar ln da razão entre o $L$ inicial e o final.

A variável de estoque de capital $(K)$ é calculada a partir da despesa acumulada de investimento do setor público municipal e pela proxy de capital residencial total. A variação do capital residencial total entre 1991 e 2000 e a despesa média de investimento do setor público permitem a construção da variável investimento. Emprega-se a média do PIB municipal - R\$ de 2000(mil) - Deflacionado pelo Deflator Implícito do PIB nacional como uma aproximação razoável para capturar o município presente na fronteira tecnológica.

A fração investida em capital físico da renda $\left(s_{k}\right)$ é construída usando a soma do investimento residencial ao investimento médio público sobre a renda. Já a construção da fração investida em capital humano $\left(s_{b}\right)$ é obtida subtraindo anos de estudo - menos de 8 - pessoas 25 anos e mais - (\%) de 100, para que dessa forma seja possível encontrar a fração com mais de 8 anos de estudo de pessoas com 25 anos ou mais. Os dados de Valor Total dos Rendimentos recebidos, a População Residente - total - Habitante, e o PIB Municipal foram retirados da base do IBGE. Enquanto que o Capital Residencial - Total, o Capital Residencial - Urbano, o Capital Humano, Anos de estudo - menos de 8 - pessoas 25 anos e mais - (\%) e a Renda per capita foram retirados do IPEA. E por fim, do Ministério da Fazenda - Secretaria do Tesouro Nacional, obteve-se a Despesa de Capital - Investimento - Municipal - R\$.

\subsection{METODOLOGIA}

A dependência espacial indica que os valores observados de um município são influenciados pela presença dos municípios vizinhos. Para considerar as relações espaciais, parte-se de uma especificação geral SARMA (média móvel e autorregresivo espacial), descrito em notação matricial por:

$$
\begin{gathered}
y=\varphi W y+X \beta+\varepsilon \\
\varepsilon=\lambda W \varepsilon+u
\end{gathered}
$$

onde $y$ é uma variável explicada, $\varphi$ corresponde ao componente autorregressivo (que capta os efeitos de transbordamento de $y$ dos vizinhos sobre a variável dependente do município em questão), $W$ a matriz de vizinhança, $\varepsilon$ o termo de erro, $X$ as variáveis explicativas, $\beta$ o vetor de coeficientes, $\lambda$ um escalar do coeficiente do erro, e $u$ o resíduo livre de correlação espacial. A 
estimação dos parâmetros do modelo SARMA pode ser feita via máxima verossimilhança.

Em linhas gerais, a econometria espacial sugere dois tipos de modelos: autocorrelação espacial na variável dependente (defasagem espacial) ou autocorrelação espacial no erro (erro espacial). Inicialmente, aborda-se o modelo SAR (modelo autorregressivo espacial), que considera a defasagem espacial da variável dependente e adota $\lambda=0$, de modo que:

$$
y=\varphi W y+X \beta+\varepsilon \quad(+u)
$$

no qual $\varepsilon$ segue uma distribuição normal com média zero e desvio-padrão $\sigma^{2}$. O teste de significância estatística de $\varphi$ permite inferir a presença ou não de dependência espacial.

No método de erro espacial (SEM), adota-se $\varphi=0$, e:

$$
\begin{gathered}
y=X \beta+\varepsilon \\
\varepsilon=\lambda W \varepsilon+u
\end{gathered}
$$

no qual $u$ segue uma distribuição normal com média zero e desvio-padrão $\sigma^{2}$. Quando $\lambda \neq 0$, um choque em um município se espalha não só para os seus vizinhos, mas para os outros municípios ${ }^{2}$.

A estimação das equações com efeito espacial será realizada pelo modelo SAR-KP, estimação fundamentada em Kelejian e Prucha (1999). Este estimador baseia-se no método de momentos generalizado (GMM), utilizando como instrumento as variáveis defasadas espacialmente, ou seja, a variável dos municípios vizinhos é considerada exógena para explicar a variável do município $i$ (por exemplo, ao invés de utilizar $X$ na regressão, instrumentaliza-se com a variável $W X$ ). Para correção da autocorrelação espacial e heterocedasticidade no resíduo, emprega-se uma matriz para diagonalizar os resíduos e tornar o estimador robusto (CARVALHO; ALBUQUERQUE, 2010). Assim, este estimador corrige para heterocedasticidade e auto-correlação nos resíduos, endogeneidade do lado direito da especificação, além de não exigir obrigatoriamente a condição de normalidade na distribuição dos erros.

A técnica que emprega o método de momentos (GMM), com a especificação em 2 estágios, considera a equação geral a seguir:

$$
y=\varrho W y+Y \nu+X \beta+\varepsilon
$$

2 Por isso, outra maneira é escrever $y=X \beta+(I-\lambda W)^{-1} u$. 
onde $y$ é um vetor coluna contendo as $n$ observações empilhadas para a variável resposta, $\varrho$ é o coeficiente do lag espacial da variável resposta, $W$ é uma matriz de vizinhança, $Y$ é uma matriz com regressores endógenos, o vetor $v$ é um vetor de coeficientes dos regressores endógenos, $X$ é uma matriz com os regressores exógenos, o vetor $\beta$ é o vetor com coeficientes dos regressores exógenos, o vetor $\varepsilon$ é um vetor coluna, de dimensão $n \times 1$ com os resíduos do modelo. Escrevendo-se a Equação (10) de forma mais concisa, temos que a variável dependente passa a ser representada por $Y$ e o regressor por $Z$, logo a regressão de interesse é:

$$
Y=Z \gamma+\varepsilon
$$

Entretanto, se $Z$ é endógeno, o estimador de $\gamma$ é inconsistente, então é preciso que se utilize instrumentos que sejam supostamente exógenos, considerando o caso de $\mathcal{Q}$ (matriz de variáveis instrumentais - VI) ser o instrumento. Os instrumentos para a variável endógena $W y$ são dados pelos lags espaciais dos regressores exógenos $W X$. Então, faz-se a regressão de $Q$ explicando Z:

$$
Z=Q \beta+\varepsilon
$$

Esta é a regressão para obtermos o $Z$ explicado, que seria não correlacionado com o $\varepsilon$ e, por isso, exógeno. Assim, o $1^{\circ}$ estágio é dado por (12). E o segundo por:

$$
Y=\hat{Z} \gamma+\varepsilon
$$

A presença de dependência espacial e a sua forma adequada são testadas. Os testes se baseiam em estimar os coeficientes pelo método dos mínimos quadrados ordinários (MQO) e testar se a omissão da componente espacial gera correlação espacial nos resíduos da regressão. Um teste para dependência espacial é realizado através da estatística I de Moran.

\section{RESULTADOS}

A Tabela 1 apresenta os resultados obtidos da estimação do modelo de crescimento incondicional, o de Solow-Swan (1956) e o estendido por Mankiw, Romer e Weil (1992). A estatística do teste de I de Moran rejeitou a hipótese nula de ausência de correlação espacial. Assim, o estimador SAR de Kelejian-Prucha (KP) parece ser o mais indicado, em comparação com o 
estimador MQO desconsiderando a relação espacial ${ }^{3}$.

$\mathrm{Na}$ Tabela 1, inicialmente discute-se os resultados por MQO nas colunas de 1 a 3 e por SAR de KP de 4 a 6 . No modelo de crescimento incondicional, os sinais dos coeficientes associados à variável explicativa seguem a expectativa, além de serem extremamente significativos estatisticamente. A estimativa por MQO apresenta um viés positivo para o coeficiente associado à renda per capita inicial; tal indício é observado em outras variáveis e será questionado mais a frente. Observou-se também que a taxa de convergência é superior na estimativa por SAR de KP, quando comparado ao obtido por MQO.

Para o modelo de Solow, o coeficiente associado à variável explicativa da renda per capita inicial $\left(y_{0}\right)$ se reduz, e ao inserir a taxa de crescimento da população ${ }^{4}$ e o capital físico, notase uma considerável diminuição na taxa de convergência entre os municípios em relação ao modelo incondicional. Mesmo padrão é encontrado nos estimados por SAR de KP, divergindo apenas quanto à magnitude, sendo os sinais encontrados os mesmos. O coeficiente associado à variável de taxa de crescimento da população apresenta o sinal negativo em ambas as estimações, ou seja, uma maior taxa de crescimento da população impacta negativamente o crescimento da renda per capita. Este mesmo coeficiente estimado por MQO apresentou efeito inferior do obtido por GMM, enquanto o coeficiente associado ao investimento em capital físico apresenta sinal positivo, tanto por GMM como por MQO. Em ambos os modelos apresentados observase, em geral, um viés positivo dos coeficientes estimados por MQO.

TABELA 1 - RESULTADOS PARA OS MODELOS DE CRESCIMENTO INCONDICIONAL, SOLOW E MRW ESTIMADOS POR MQO E GMM (SAR DE KELEJIAN E PRUCHA)

\begin{tabular}{|c|c|c|c|c|c|c|}
\hline \multirow{2}{*}{ Variáveis } & \multicolumn{3}{|c|}{ Mínimos Quadrados Ordinários } & \multicolumn{3}{|c|}{ GMM (SAR - KP) } \\
\hline & Incondicional & Solow & MRW & Incondicional & Solow & MRW \\
\hline \multirow{2}{*}{ Constante } & 0.6047 & 0.3347 & 0.4319 & 0.6740 & 0.4373 & 0.5396 \\
\hline & 0.00 & 0.00 & 0.00 & 0.00 & 0.00 & 0.00 \\
\hline \multirow[t]{2}{*}{ Renda inicial } & -0.0556 & -0.0159 & -0.0846 & -0.0702 & -0.0302 & -0.0989 \\
\hline & 0.00 & 0.00 & 0.00 & 0.00 & 0.00 & 0.00 \\
\hline \multirow{2}{*}{$\ln (n+g+\delta)$} & & -0.1110 & -0.1124 & & -0.0926 & -0.0936 \\
\hline & & 0.00 & 0.00 & & 0.00 & 0.00 \\
\hline \multirow[t]{2}{*}{ Capital físico } & & 0.0891 & 0.0889 & & 0.0822 & 0.0827 \\
\hline & & 0.00 & 0.00 & & 0.00 & 0.00 \\
\hline \multirow[t]{2}{*}{ Capital humano } & & & 0.0868 & & & 0.0861 \\
\hline & & & 0.00 & & & 0.00 \\
\hline Taxa de convergência & 0.6358 & 0.1785 & 0.9822 & 0.81 & 0.34 & 1.16 \\
\hline$\lambda$ & & & & 0.2466 & 0.1843 & 0.1799 \\
\hline Residual variance (sigma squared) & & & & 0.0420 & 0.0401 & 0.0385 \\
\hline GM argmin sigma squared & & & & 0.0423 & 0.0403 & 0.0387 \\
\hline $\mathrm{F}$ & 151.90 & 175.60 & 193.80 & & & \\
\hline$p$-valor & 0.0 & 0.00 & 0.00 & & & \\
\hline
\end{tabular}

3 Adicionalmente, resultados obtidos pelo estimador espacial SARMA foram utilizados, mas omitidos, sendo o estimador preterido por não corrigir a heterocedasticidade, autocorrelação nos resíduos e para endogeneidade.

4 Como em $(n+g+\delta)$ tanto $g$ quanto $\delta$ são constantes, tomou-se a liberdade de tratá-lo como taxa de crescimento da população apenas para efeito de simplificação. 
TABELA 1 (CONTINUAÇÃO) - RESULTADOS PARA OS MODELOS DE CRESCIMENTO INCONDICIONAL, SOLOW E MRW ESTIMADOS POR MQO E GMM (SAR DE KELEJIAN E PRUCHA)

\begin{tabular}{l|rrrr|r|r}
\hline \multirow{2}{*}{ Variáveis } & \multicolumn{2}{|c|}{ Mínimos Quadrados Ordinários } & \multicolumn{2}{c}{ GMM (SAR - KP) } \\
\cline { 2 - 6 } & Incondicional & Solow & MRW & Incondicional & Solow & MRW \\
\hline I de Moran & 14.19 & 9.74 & 9.48 & \\
p-valor & $\mathbf{0 . 0 0}$ & $\mathbf{0 . 0 0}$ & $\mathbf{0 . 0}$ & \\
LM-erro & $\mathbf{0 . 0 0}$ & $\mathbf{0 . 0 0}$ & $\mathbf{0 . 0 0}$ \\
Robusto LM-erro & $\mathbf{0 . 0 0}$ & $\mathbf{0 . 4 4}$ & $\mathbf{0 . 8 0}$ \\
LM-lag & $\mathbf{0 . 0 0}$ & $\mathbf{0 . 0 0}$ & $\mathbf{0 . 0 0}$ \\
Robusto LM-lag & $\mathbf{0 . 0 0}$ & $\mathbf{0 . 0 0}$ & $\mathbf{0 . 0 0}$ & \\
\hline
\end{tabular}

FONTE: Elaboração própria.

NOTA: * Os elementos que estão em negrito são os $p$-valores; ${ }^{* *} \mathrm{O} \lambda$ indica a intensidade da autocorrelação espacial entre os resíduos da equação observada; ${ }^{* * *}$ As taxas de convergência foram calculadas seguindo a fórmula utilizada por Pede, Florax e Groot (2008), $100 \ln (b+1) /-T$; onde $b$ é o coeficiente de $y_{0}$ e $T$ é o período de tempo utilizado.

De acordo com o modelo MRW pela inserção do capital humano, todos os coeficientes associados às variáveis explicativas apresentam os sinais esperados e são estatisticamente significativos. Entretanto, a magnitude do coeficiente da variável renda per capita $\left(y_{0}\right)$ eleva-se, em módulo, ao incluir a variável de capital humano. Verifica-se um elevado crescimento na taxa de convergência entre os municípios, quando os modelos de Solow e MRW são comparados, embasando a crítica de MRW (1992), sobre a subestimação obtida pelo modelo de Solow. Uma pequena diminuição do papel do capital físico na taxa de crescimento dos municípios é observada na estimação via MQO, ou seja, neste caso parece que o modelo de Solow retrata a presença de uma sobrevalorização do papel do capital físico, pela omissão da variável capital humano. E quando esta mesma variável é comparada ao modelo corrigido espacialmente observa-se, em média, uma sobrevalorização entre as estimações.

O curioso é que ao observar apenas os modelos de Solow e de MRW estimado pelo SAR de KP, é possível notar que a inserção do capital humano, que no MQO vinha acompanhada de pequena queda no coeficiente de capital físico e aumento na magnitude da renda, agora vem acompanhada de um leve aumento no coeficiente do capital físico. Pode-se perceber que o coeficiente associado à variável capital humano foi significativo e com sua importância como determinante do crescimento econômico podendo ser mensurada, por exemplo, pelo seu efeito na magnitude dos demais coeficientes do modelo.

\section{CONCLUSÕES}

Os resultados obtidos foram de que a presença de correlação espacial é confirmada pelo teste do I de Moran, e que os capitais físico e humano são relevantes para explicar o crescimento econômico, mesmo quando se considera a presença de correlação espacial. 


\section{REFERÊNCIAS}

MANKIW, N.; ROMER, D.; E WEIL, D. A contribution to the empirics of economic growth. Quarterly Journal of Economics, v. 107, n. 2, p. 407-37, 1992.

PEDE, V. O.; FLORAX, R. J. G. M; GROOT, H. L. F. Technological leadership, human capital and economic growth: a spatial econometric. Annales d'Économie et de Statistique, n. 87/88, p. 103-124, jul./dez. 2008.

SOLOW, R. M. A contribution to the theory of economic growth. Quarterly Journal of Economics, v. 70, n. 1, p. 65-94, 1956. 
